\title{
Simulation of MEMS based drug delivery system
}

\begin{abstract}
Transdermal drug delivery is having lot of attention in medical field. A gaining need of controlled drug delivery enabled MEMS drug delivery systems to penetrate this field. In this, paper we designed a micro-pump with piezo electric actuation scheme. The simulation studies by FEM tool provides the velocity of $0.72 \mathrm{~m} / \mathrm{s}$ and pressure by piezo material on reservoir IS $326 \mathrm{~Pa}$ and von misses stress obtained $1.93 * 10^{7} \mathrm{~N} / \mathrm{m}^{2}$.
\end{abstract}

Keywords: MEMS, bio-MEMS, COMSOL, transdermal drug delivery
Volume 2 Issue $3-2017$

\author{
Jasti Sateesh, Srinivasa Rao K \\ Department of Electronics and Communication Engineering, KL \\ University, India
}

Correspondence: Srinivasa Rao K, Department of Electronics and Communication Engineering KL University, Andhra Pradesh, India, Email srinivasakarumuri@gmail.com

Received: January 30, 2017 | Published: April II, 2017

\section{Introduction}

There is a plenty of room at the bottom stated by Richard Feynman. If we decrease the size of the device, we can incorporate different devices onto a single chip. Miniatured devices is a basic demand by the society for their comfort and ease of use. The miniaturization in all aspects is a growing requirement. Micro Electro Mechanical Systems (MEMS) is a technology, by which a complete system can be made, that works for a specific operation in micro size. Bio-MEMS the interdisciplinary nature of MEMS that mainly focuses on Bio species, their reacting compounds, and their detection. Already plenty of research has done in Bio-MEMS, brought out sensors as glucose sensors, ${ }^{1}$ PD detection, ${ }^{2} \mathrm{~TB}$ detection ${ }^{3}$ many more. MEMS technology has been applied to the drug delivery systems, because of its high application range. This technology termed as micro fluidics, that deals with fluid flow physics in micro level.

Conventional ways of drug delivery such as tablets, capsules and injections do not deliver the drug in an efficient way. There is no control over the drug delivery range. The subcutaneous injections pierce the skin and may cause damage to the nerves, tissues and arteries. It is estimated that $20 \%$ of world population is having trypanophobia (fear of injection). ${ }^{4}$ The tablets are manufactured with some ingredients to give colour, avoid friction and dissolve in stomach much easily, by these extra fittings there may be mall functioning of the drug. The drug that is delivered to the body will have maximum and minimum limits. The maximum limit above which the drug is toxic, the minimum limit below with no benefit of supplying the drug to body. MEMS based drug delivery systems mainly consisting of drug reservoir, micropump, micro-valve, and micro needle. ${ }^{5}$ Drug reservoir for storage of drug, micro-pump is to collect the drug from reservoir and pump it out with a force that equal to enter the body and micro needle for piercing the skin and allowing the drug to enter the body with some flow rate. The heart of MEMS DDS is micro pump. Piezo electric micro pumps have gaining interest because of its numerous advantages like broad range of fluids can be pumped and can be fabricated with silicon micro-machining process. Magnetic DDS using magnetic field gradient proposed in, ${ }^{6}$ the fabrication of magnets is very much tough task in micro size. Electro kinetics localized effects which controls the release of drugs. ${ }^{7}$ Micro pump designed using SU-8 and PDMS membrane using nozzle type of structure has illustrated in. ${ }^{8}$ Heating of resistive material beneath the drug reservoir, which in turn produces bobbles to push the liquid in the reservoir out. The different types of designs and future of micro needles are given in. ${ }^{8}$ The innovative double moulding of the needle at the ends the holes are on either side. ${ }^{10}$

The designed micro pump is based on piezo electric actuation technique, the drug reservoir is designed with PDMS material beneath of this layer is a piezo electric layer (PZT-5A) and electrodes with aluminium are designed. The width is $500 \mathrm{um}$, length is $1400 \mathrm{um}$ and the height is $300 \mathrm{uum}$. Wall material is silicon dioxide. Inlet width (100um) is larger than outlet (50um), for increasing the flow rate (Figure 1). The simulations are carried out by FEM tool COMSOL Multiphysics. The inlet pressure is assumed to be as $0.1 \mathrm{~Pa}$ and the output velocity is $0 \mathrm{~m} / \mathrm{s}$. the wall is assumed to be with slip. When an input voltage applied the piezo-electric material changes its shape and exerts a pressure on the reservoir. By on and off of the input voltage we can get the pumping operation The obtained results are as shown Figure 2. The flow rate of $0.72 \mathrm{~m} / \mathrm{s}$ is obtained as the more red lines are at the outlet of the device, the flow rate very high at the outlet. The exerted by the pizo material is obtained as $326 \mathrm{~Pa}$ as shown in Figure 3. The vonmises stress experienced by the structure is $1.93 * 10^{7} \mathrm{~N} /$ $\mathrm{m}^{2}$. The results clearly show that output velocity obtained is perfectly useful for the use in drug delivery.

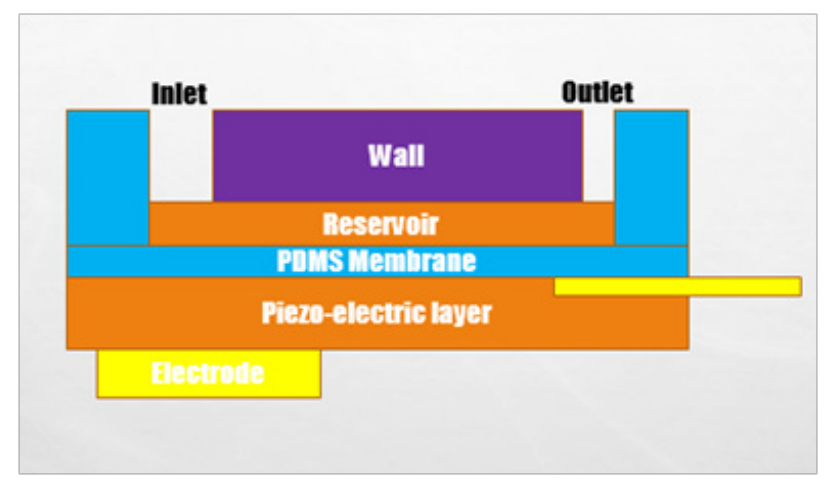

Figure I Schematic Structure of the micro-pump.

In this paper the modelling and simulation of micro pump for micro drug delivery system is defined and simulated. Analysis of obtained results is presented. The results show that this device can perfectly fit for the micro-pumping in DDS. The growing demand of MEMS based drug delivery systems can be accompanied by this model. The future of this work is to vary the design for different materials to decrease the power consumption, fabrication cost and simplified design. 


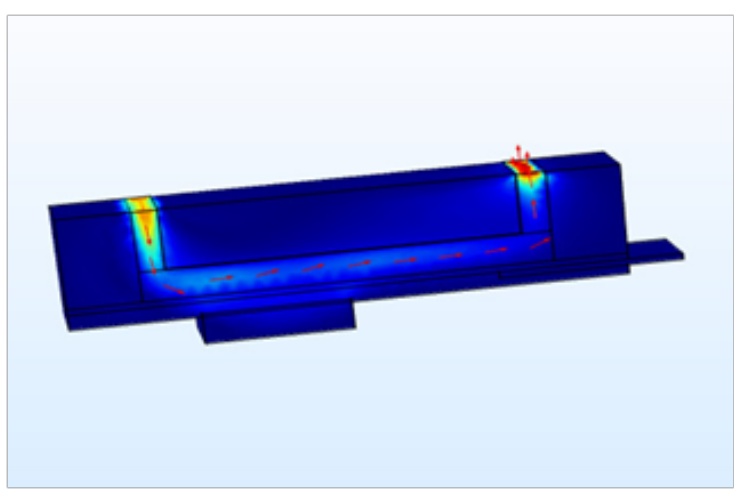

Figure 2 Simulated results of outflow velocity and Pressure exerted.

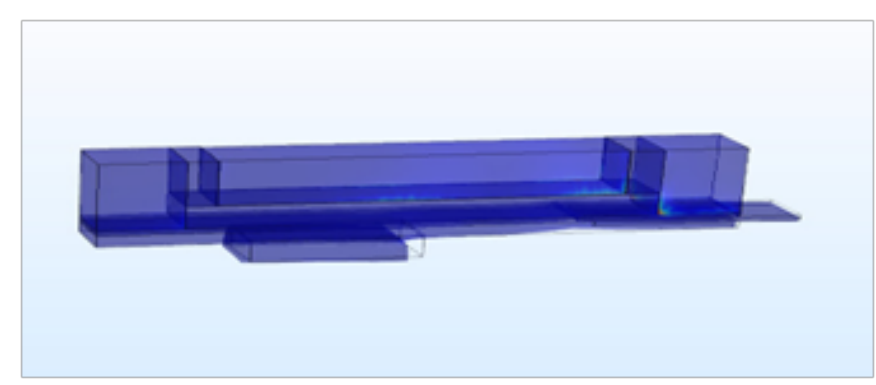

Figure 3 Vonmises stress obtained.

\section{Acknowledgements}

None.

\section{Conflict of interest}

The author declares no conflict of interest.

\section{References}

1. Jasti Sateesh. Design of MEMS bio-sensor for glucose measurement. Journal of mechanics and MEMS. 2016;8(1):83-89

2. Prasad GRK. Micro electro mechanical system based sensor for early detection of parkinson's disease-design and simulation. Int J Pharm Bio Sci. 2017;8(1):390-395.

3. Srinivasa Rao K. Design and analysis of MEMS based bio sensor for TB detection. 2016 international conference on electrical, electronics; India: 2016.

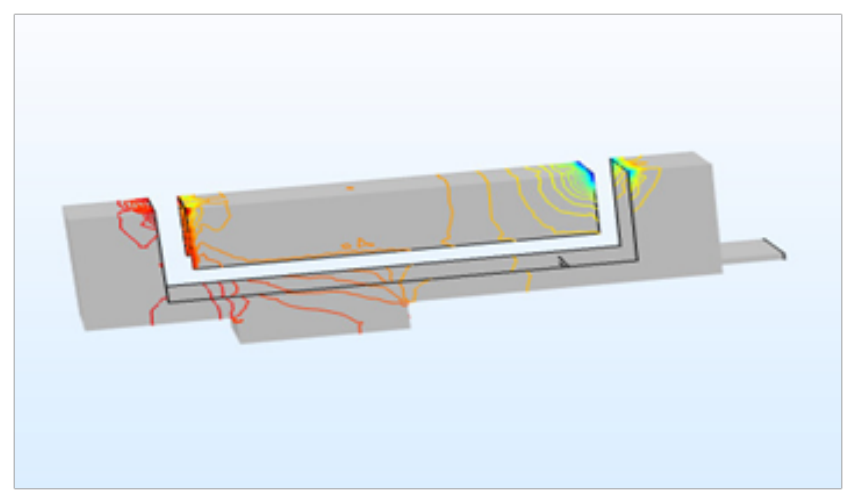

4. Wright S, Yelland M, Heathcote K, et al. Fear of needles-nature and prevalence in general practice. Aust Fam Physician. 2009;38(3):172176.

5. Samajdar DP, Mallick D, Bhattacharyya A. Design, simulation and study of micro-pump, Micro-valve and Micro-needle for biomedical applications. Expert from the proceedings of the 2012 COMSOL conference in Bangalore; India: University of Calcutta; 2012.

6. Xiaotao Han, Quanliang Cao, Liang Li. Design and evaluation of threedimensional electromagnetic guide system for magnetic drug delivery. Ieee Transactions on Applied Superconductivity. 2012;22(3):44014044401404.

7. Aram J Chung, Donn Kim, David Erickson. Electrokinetic microfluidic devices for rapid, low power drug delivery in autonomous Microsystems. Lab Chip. 2008;8:330-338.

8. Bidhan Pramanick, PK Dey, Soumen Das, et al. Design and development of a PDMS Membrane based SU-8 Micropump for drug delivery system. Institute of Smart Structures and Systems (ISSS). 2013;2(1):1-9.

9. Danny Jian Hang Tng, Rui Hu, Peiyi Song, et al. Approaches and challenges of engineering implantable microelectromechanical systems (MEMS) drug delivery systems for In Vitro and In Vivo applications. Micromachines. 2012;3(4):615-631.

10. Marion Sausse Lhernould, A Delchambre. Innovative design of hollow polymeric microneedles for transdermal drug delivery. Microsyst Technol. 2011;17(10-11):1675-1682. 